\title{
Springy Material Boosts Projectile Performance
}

\section{Adding a soft, springy material to a solid projectile leads to a threefold increase in kinetic energy when the projectile is launched upward.}

\author{
By Philip Ball
}

$\prod$ hrowing is as central to some sports today as it once was for hunting and warfare. A team of scientists now shows how throwing efficiency could be improved by engineering the elasticity of the projectile [1]. Their experiments show that, compared with a rigid projectile, one that includes some soft, springy material can receive up to 3 times more kinetic energy when launched upward. The results could be used to design better projectiles in sports and could lead to shoe designs that enhance energy transfer during walking and running.

Throwing involves a complex chain of events that couple the movements of the limbs, skeleton, muscles, and tendons to give the projectile kinetic energy. These movements are not driven purely by muscle power but also involve the release of elastic energy stored in the tendons, as in a catapult.

Franck Celestini and Christophe Raufaste of the University of Côte d'Azur, France, and their colleagues have now looked at how that energy transfer might be improved in a simple model system in which a projectile is launched from an upward-moving platform. They have previously shown that timing can be crucial to efficient propulsion when the projectile is deformable and elastic. A drop of water launched upward from an oscillating platform can gain up to 2.5 times the kinetic energy of a rigid ball with identical mass when the platform vibrates at about one-third the frequency of the drop's intrinsic oscillations [2] (see also Focus: Superpropulsion of Liquid Drops).

The team has now run similar experiments on rigid objects with an added elastic component as a way of studying throwing efficiency in a simplified system. The researchers used a spring-loaded platform to deliver upward thrust to cylindrical projectiles $12 \mathrm{~mm}$ wide and $15 \mathrm{~mm}$ tall. The rigid portion of each cylinder was made of hard plastic, and the researchers added a squishy layer of gelatin hydrogel of various thicknesses (from about $6 \mathrm{~mm}$ to the full height) to the bottom of some projectiles. They determined the efficiency of energy transfer by measuring the heights to which the projectiles rose.

The researchers found that a soft layer can always improve the ejection efficiency relative to a rigid cylinder. The optimal performance occurs when the duration of the platform's upward acceleration is approximately equal to the natural oscillation period of the vibrating soft layer, so that the most energy can be transferred from platform to projectile. The best improvements were for soft layers of up to about 30\% of the total cylinder height-where the efficiency could be improved threefold. The enhancement factor remained around 2.5, however, when the whole projectile was soft.

In some ball sports, the difficulty of transferring elastic energy efficiently from the arm to the ball can cause much of the kinetic energy of the throw to stay within the body, potentially leading to injury. Such overexertion is a common problem for baseball pitchers, for example. Celestini and colleagues say that in such cases, giving the ball a more elastic layer at the trailing edge could greatly boost the power of the throw while exposing the player to a lower risk of injury.

They also believe that their approach might be used to improve energy transfer in running shoes. "We think that the soft elastic sole plays the same role as the soft layer in the projectile," says Raufaste. He says that reaching the highest efficiency would require customizing the shoe for each runner. 

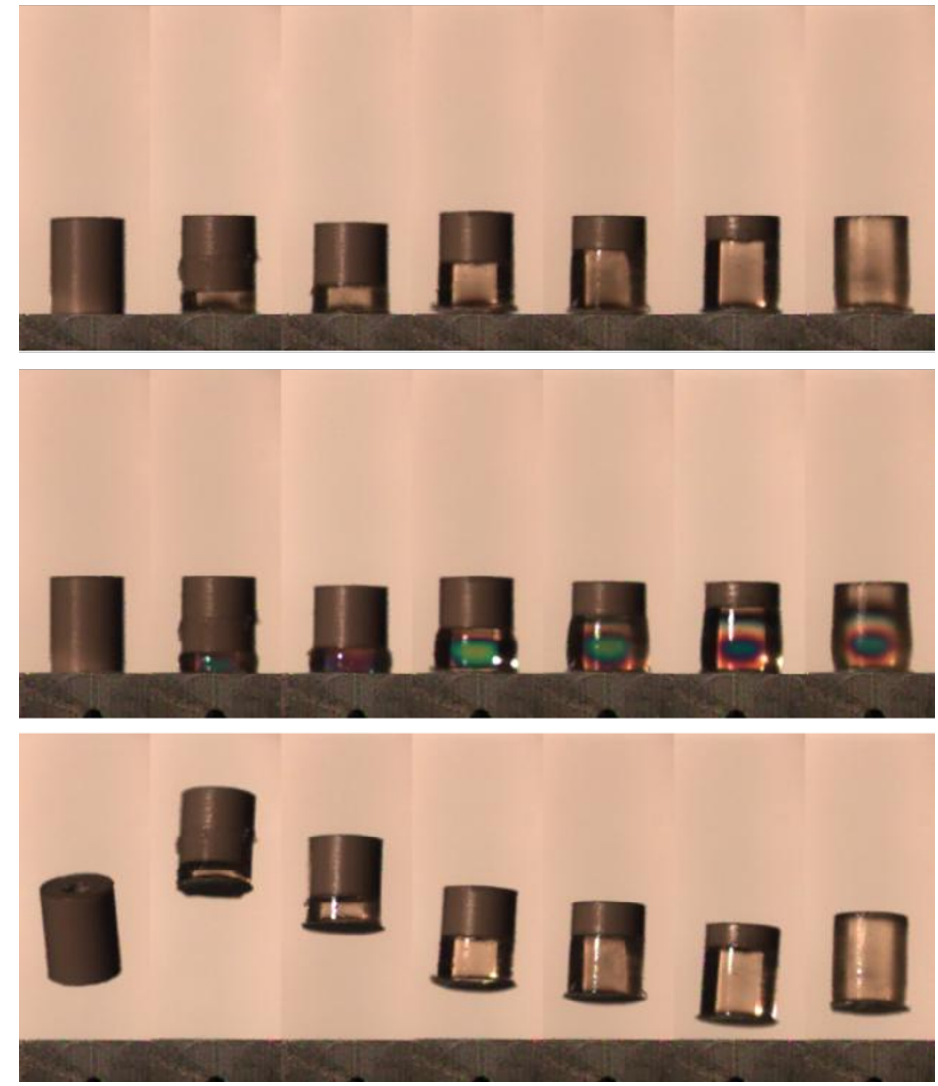

Launch sequence. A lineup of plastic projectiles is propelled upward by an accelerating platform, with each cylinder having a different thicknesses of a gel layer at its base. The projectiles are shown before release (top), during deformation as the platform rises (middle), and at their maximum heights (bottom). (See video below.)

Credit: F. Celestini et al. [1]

The new work is reminiscent of a simple jumping robot built in 2012 that incorporated a spring, says mechanical engineer Madhusudhan Venkadesan of Yale University [3] (see also Focus: Robot Shows How to Jump High). The new results provide "a nice demonstration of the central idea that the elastic member should not be too soft or too stiff in order to benefit from its mechanics," he says. The paper "could add to other efforts to realize jumping robots or toys that capitalize on internal elastic modes."
Brandon Erickson of Rush University Medical Center, Illinois, a specialist in orthopedic surgery and sports medicine, warns that this simple setup doesn't capture the complex

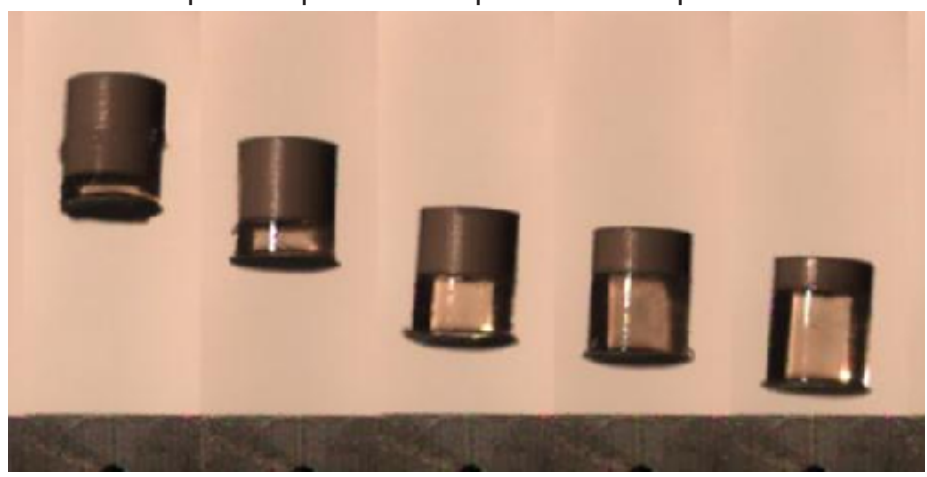

Projectiles with various thicknesses of gel (transparent layer) launched in a single oscillation cycle from a platform moving at a frequency of $76 \mathrm{~Hz}$. The transient dark regions in the gel layer are caused by changes in optical properties due to compression, which provides a view of the gel's internal oscillations. The best improvement in efficiency of energy transfer occurs for thinner gel layers. (The gel thickness determines the platform oscillation frequency at which each projectile reaches its maximum height.) Credit: F. Celestini et al. [1]

biomechanics of real throwing-a point that Celestini and colleagues acknowledge. Besides, Erickson says, it's not clear that a large enhancement in efficiency would always be useful in sports. If a baseball were designed to be thrown much faster than today's fastballs, it could be impossible to hit and could pose a hazard for the catcher.

Philip Ball is a freelance science writer in London. His latest book is How To Grow a Human (University of Chicago Press, 2019).

\section{REFERENCES}

1. F. Celestini et al., "Contact layer as a propelling advantage in throwing," Phys. Rev. Applied 14, 044026 (2020).

2. C. Raufaste et al., "Superpropulsion of droplets and soft elastic solids," Phys. Rev. Lett. 119, 108001 (2017).

3. J. Aguilar et al., "Lift-off dynamics in a simple jumping robot," Phys. Rev. Lett. 109, 174301 (2012). 